\title{
Modelling vaccination strategies against foot-and-mouth disease
}

\author{
M. J. Keeling ${ }^{\star}$, M. E. J. Woolhouse $†$, R. M. May $\ddagger$, G. Davies $\$$ \& B. T. GrenfelI||
}

${ }^{*}$ Department of Biological Science \& Mathematics Institute, University of Warwick, Gibbet Hill Road, Coventry, CV4 7AL, UK

$\dagger$ Centre for Infectious Diseases, College of Medicine and Veterinary Medicine, University of Edinburgh, Easter Bush, Roslin, Midlothian, EH25 9RG, UK

$\ddagger$ Department of Zoology, University of Oxford, Oxford, OX1 3PS, UK

$\$$ Zinnia, Kettlewell Hill, Woking, Surrey, GU21 4JJ, UK

II Department of Zoology, University of Cambridge, Downing Street, Cambridge, CB2 3EJ, UK

Vaccination has proved a powerful defence against a range of infectious diseases of humans and animals. However, its potential to control major epidemics of foot-and-mouth disease (FMD) in livestock is contentious. Using an individual farm-based model, we consider either national prophylactic vaccination campaigns in advance of an outbreak, or combinations of reactive vaccination and culling strategies during an epidemic. Consistent with standard epidemiological theory, mass prophylactic vaccination could reduce greatly the potential for a major epidemic, while the targeting of high-risk farms increases efficiency. Given sufficient resources and preparation, a combination of reactive vaccination and culling might control ongoing epidemics. We also explore a reactive strategy, 'predictive' vaccination, which targets key spatial transmission loci and can reduce markedly the long tail that characterizes many FMD epidemics. These analyses have broader implications for the control of human and livestock infectious diseases in heterogeneous spatial landscapes.

The epidemic of FMD in 2001 exerted a major toll on Great Britain's livestock industry and highlighted a series of important questions about the management of infectious diseases in animal populations ${ }^{1}$. Detailed records of this epidemic ${ }^{2}$ have allowed the development of epidemiological models that capture the main features of epidemic spread in space and time, and the impact of control strategies $^{3-7}$.

After the introduction of livestock movement restrictions on 23 February 2001, the epidemic showed intense local stochastic spread, together with rarer long-distance jumps ${ }^{2,3,7}$. This generated a severe initial epidemic in localized regions, followed by a long, irregular epidemic tail. Control measures used during the 2001 FMD epidemic were movement restrictions, biosecurity and culling, coupled with surveillance. Different models all indicated that prompt culling of infected premises (IPs) and at-risk farms (dangerous contacts, DCs, and contiguous premises, CPs) was necessary to control the disease because of the intense local spread ${ }^{3,5-7}$. Although reactive vaccination of livestock during the epidemic was proposed, various biological and logistical problems confounded its likely effectiveness (see Supplementary Information). Here, we consider the potential impact of both reactive and prophylactic vaccination ${ }^{8,9}$ on future FMD epidemics, in terms of expected epidemic size and duration, for a variety of models. We concentrate on cattle vaccination, but also compare this default with vaccinating other livestock species. The fine spatial grain of the British data also allows us to explore two general epidemiological questions. First, how well does the performance of vaccination in this complex spatial epidemic match the predictions of standard theory ${ }^{8}$ ? Second, can we use our detailed spatial model to generate predictive vaccination strategies, which optimize disease control by targeting key 'nodes' in the spatial transmission network?

To model the dynamics of vaccination, we need to allow for the biological limitations of current FMD vaccine formulations. Major determinants of the effectiveness of prophylactic vaccination are uptake (the proportion of animals vaccinated) and efficacy (the proportion of vaccinated animals that are protected $)^{10-12}$. Efficacy (typically no more than $90-95 \%{ }^{13}$ ) can be confounded by antigenic variation between FMD virus strains, requiring some prior assessment of which strains present the greatest threat. Reactive vaccination evades the problem of strain uncertainty but suffers from a significant delay (around 4 days for high-potency vaccines and 10 days for standard formulations ${ }^{14}$ ) before vaccinated animals are protected $^{7}$.

To focus the analysis, simulations are based on the highly disseminated 2001 epidemic in Great Britain as a pessimistic model; however, we interpret these results in terms of the generic performance of future vaccination campaigns, where appropriate stocks of highly effective vaccines and strategies for delivering them are in place before the start of the epidemic. We address these issues using a relatively parsimonious individual-based stochastic model (see Methods), which accurately captures the overall spatiotemporal pattern of the 2001 epidemic in Great Britain ${ }^{7}$ (see Supplementary Information for a discussion of parameter sensitivity and model assumptions).

\section{Prophylactic vaccination}

Figure 1 reports the impact of prophylactic vaccination under a variety of assumptions about the use and characteristics of the vaccine. Even with vaccination, infected and at risk farms would still be likely to be culled ${ }^{15}$; we therefore take as our baseline model vaccination of cattle, combined with culling of IPs and epidemiologically identified DCs arising from them ${ }^{7}$. The success of the strategy is defined by the size of the epidemic as a function of the number of farms or animals successfully vaccinated before the outbreak starts (Fig. 1a). The simplest case is vaccination of cattle on a given number of farms chosen at random irrespective of location, size or other risk factors (red line). The resulting number of cases drops relatively rapidly with the number of vaccinated farms; vaccinating about 25,000 cattle farms reduces the size of the epidemic to that achieved by stringent IP, DC and CP culling ${ }^{7}$ (dashed horizontal line). Vaccinating above 80,000 farms prevents almost all major epidemics although, due to the stochasticity of the epidemic process, a precise threshold is not determinable.

Figure $1 \mathrm{~b}$ considers the same models as Fig. 1a, but shows the expected duration of the epidemic. High vaccine uptake significantly reduces the length of the outbreak by breaking chains of transmission, thus preventing percolation of the infection. This is in contrast with the results of non-spatial deterministic and stochastic 
models (see Supplementary Information and Box 1).

These results assume high vaccine efficacy (90\%); however, as efficacy may vary considerably, it is important to assess the implications of lower values ${ }^{14}$. The inset to Fig. 1a considers vaccine efficacies of 90, 70 and 50\% - if we express vaccine uptake in terms of the number of animals protected rather than the number of farms, the resulting epidemic size and duration (not shown) are largely independent of efficacy. This result is similar to standard theoretical predictions ${ }^{10,11,16}$; essentially, we can compensate for low efficacy by increasing uptake and thereby maintaining the number of animals protected. Because our model epidemics tend to last less

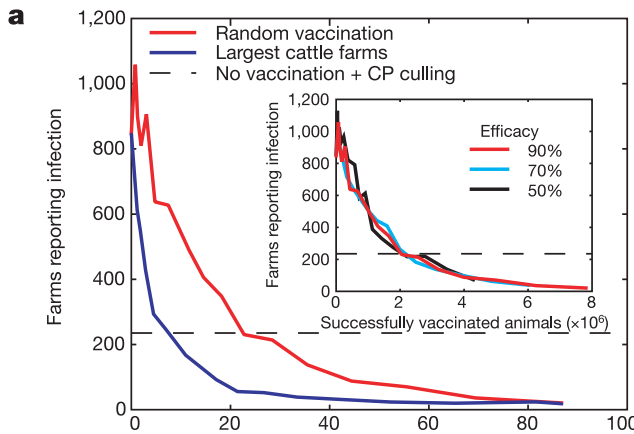

b

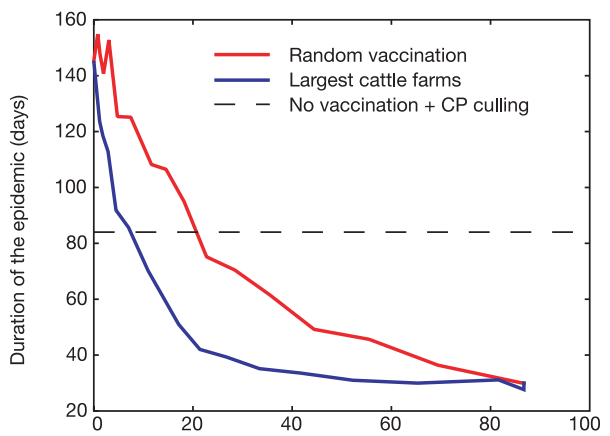

c

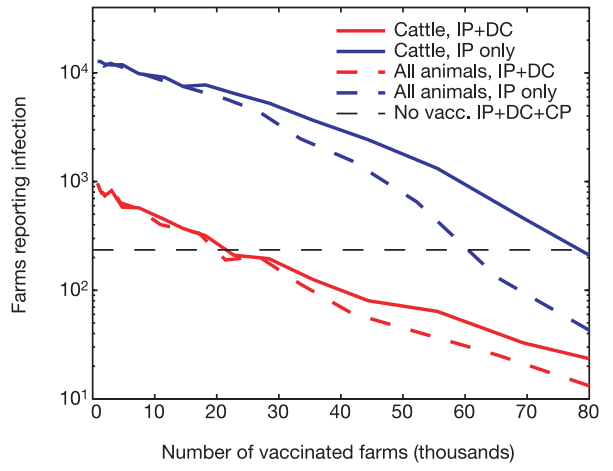

Figure 1 The fate of an introduction of foot-and-mouth disease (FMD) into five randomly chosen farms in Britain after mass vaccination. Infected premises (IP) and dangerous contact (DC) culls (averaging one DC per IP) were used to control the epidemics, and movement restrictions were enforced two weeks after the arrival of the disease. A comparison is made to the case where contiguous premises (CP) culling $(70 \%$ of CPs are culled) is used instead of vaccination. (All results are the average of 500 simulations, in many of which the epidemic fails to take off.) a, Expected number of farms reporting infection against the number of farms vaccinated. Only cattle are vaccinated and the efficacy is assumed to be $90 \%$. The inset figure compares three levels of efficacy for random vaccination. $\mathbf{b}$, Expected duration of the epidemic against number of farms vaccinated. The same three vaccination strategies as in a are used, vaccinating cattle with $90 \%$ efficacy. c, Expected number of farms reporting infection when farms are vaccinated at random. Dashed lines indicate that all animals are vaccinated against FMD, whereas solid lines correspond to vaccinating cattle only. Red curves indicate when both IP and DC culls are performed; blue curves are when only IP culls are used. than 6 months, we do not model explicitly the problem of duration of protection provided by vaccination (Supplementary Information).

Figure 1c explores a range of other prophylactic vaccination options and epidemiological models. First, we consider vaccination of sheep and pigs as well as cattle; this would be more difficult in practice but is useful to explore in terms of the impact of herd immunity. Unsurprisingly, vaccinating all three species (dashed line) produces smaller epidemics than vaccinating cattle only; however, in terms of the total numbers of animals vaccinated, concentrating on cattle alone is much more efficient. Further simulations (see Supplementary Information) indicate that vaccinating sheep only is not an efficient strategy for the FMD strain responsible for the 2001 epidemic in Great Britain, requiring approximately three times as many animals to be vaccinated to achieve the same effect. Sheep-only vaccination also generates a much more variable outcome, as there remains a significant probability of major cattle epidemics (Supplementary Information). Standard theory (Box 1) indicates that targeting of high-risk groups can significantly increase the efficiency of vaccination ${ }^{8,10,17}$. This is also true here: large cattle farms are particularly susceptible to FMD infection ${ }^{6,7}$ and targeting them (see Methods) significantly decreases the size of the epidemic for a given level of vaccine uptake. In practice, regional targeting of control, informed by risk maps ${ }^{7}$, would probably further improve this performance.

For all-species vaccination, a major epidemic is prevented at around $30 \%$ vaccine uptake (40,000 farms). This equates roughly to the results of simple deterministic models ${ }^{8}$, which predict that the effective reproductive ratio, $R$, of 1.45 after movement restrictions ${ }^{4}$ would require $31 \%(=1-1 / R)$ protection to prevent a major epidemic, given that basic FMD control measures are implemented as well. Figure 2 explores this correspondence further by plotting the standard vaccination threshold ${ }^{8}$ against results from the full simulations. The analytical approximation (dashed line) captures the basic shape of the curve calculated for the full system (red) reasonably well; again targeting vaccination at cattle only on large cattle farms (blue) proves more efficacious. A more detailed comparison of epidemics generated by spatial simulations and a simple structured model is given in Box 1. The correspondence between these models is revealing - the deterministic approximation captures the essential shape of the spatial stochastic epidemic, but there is some overestimation of cases during the initial decline of the epidemic. This discrepancy is due to the spatial correlation of high-risk cattle farms, so that vaccination has a larger than anticipated impact in the already infected areas. The tail of the outbreak relies on occasional long-distance jumps to new susceptible areas, and so is underestimated by the non-spatial model; however, the overall shape of the epidemic is partly determined by interspecies heterogeneities in transmission.

Efficient DC culling superimposed on vaccination is important in terms of reducing the epidemic (Fig. 1c, comparison between red and blue lines). This is similar to the general result that prompt local detection and elimination of potential infection is a vital aspect of disease control ${ }^{3,4,6,7,18}$. Finally, we have so far focused on the epidemiological situation in the 2001 epidemic of Great Britain where pigs were not heavily involved; however, this is frequently not the case ${ }^{19,20}$. An increased level of pig transmission-reflecting the very high levels of aerosol spread from pigs infected with certain strains of $\mathrm{FMD}^{21}$ — could result in a much larger epidemic that requires far higher levels of vaccination to control (Supplementary Information). This illustrates the importance of considering a range of infection parameters and the risk of basing strategic planning on the outcome of a single epidemic model.

\section{Reactive vaccination}

We consider three approaches to reactive vaccination. First, we assess the feasibility of rapidly achieving herd immunity after 
detection of an outbreak-mass reactive vaccination. If this is not possible, we explore more limited strategies of neighbourhood vaccination: ring vaccination, where vaccination is targeted locally in a ring around identified sources of infection ${ }^{22}$; and predictive vaccination, where farms that are expected to contribute most to future spatial transmission of infection are targeted.

Of the three approaches to reactive vaccination, we focus on the most likely and most epidemiologically efficient model: mass vaccination of cattle, coupled with IP and DC culling. After the detection of an FMD outbreak, control is governed by two logistical constraints: (1) the ensuing delay before vaccination can be started; and (2) the number of animals that can be vaccinated per day (see Supplementary Information). We assume that sufficient mobilization of vaccine production and veterinary effort could allow vaccination to begin within a week and reach substantial proportions of Great Britain's cattle herd within days or a few weeks after that (see Supplementary Information). We adopt these estimates as the best case and also explore a more pessimistic set of longer pre-vaccination delays and slower subsequent vaccination rates. Vaccination is applied nationally, but to improve efficiency the largest cattle farms are targeted first (see Methods).

Figure 3a (solid lines) depicts the average size of the simulated epidemic, which declines rapidly with daily vaccination rate, reaching a lower plateau at a rate of around 300,000 cattle per day. This rate corresponds to achieving the deterministic vaccination threshold in around 25 days. The delays, which subsume the time between detection of cases and delivery of vaccine and between vaccination and protection, also have a significant effect on vaccine effectiveness (Fig. 3a); increasing lags allow progressively more of the epidemic to 'escape' the campaign and therefore raise the epidemic size reached even at very high levels of vaccine uptake. Figure $3 \mathrm{~b}$ (solid lines) shows the corresponding impact of mass reactive vaccination on the duration of the epidemic. Essentially, high levels of herd immunity in cattle can prevent the long tail of the epidemic. Note that relatively short initial delays (less than one month) do not destroy this effect, as later levels of herd immunity

\section{Box 1}

\section{Vaccination and control in a heterogeneous environment}

Earlier work on vaccination programmes against infectious diseases of humans usually treated populations as homogeneous, and thus correctly estimated high levels of vaccination coverage as being necessary for eradication in most cases. Stimulated largely by work on HIV/AIDS, more recent work has focused on vaccination or other preventive measures in populations with substantial heterogeneity in exposure to, and/or transmission of, infection. One very general conclusion emerges from these studies: if such heterogeneous populations are incorrectly assumed to be homogeneous, then the fraction needing vaccination to eradicate infection will be underestimated (often severely) if we vaccinate at random. By contrast, the eradication fraction can be reduced (often markedly) if we vaccinate optimally, taking advantage of the heterogeneities ${ }^{8}$.

This Box sketches a simple 'toy' model, which gives some insight into the situation where culling and vaccination are combined to control an outbreak of infectious disease among livestock, in such a heterogeneous situation.

Understanding and predicting the dynamics of FMD is complicated by two forms of heterogeneities that have important but conflicting roles. Spatial heterogeneity and the local nature of transmission mean that the number of susceptibles around an infected premise is rapidly depleted. This negative spatial correlation between infected and susceptible farms will reduce the speed with which the epidemic spreads ${ }^{3,28}$. Farm-level heterogeneities arise due to the differences in number and composition of livestock. Previous analysis has indicated that large farms are both more susceptible and more transmissive than smaller farms, and that cattle farms are more susceptible than sheep farms ${ }^{7}$. This form of heterogeneity can be understood using the modelling framework developed for the heterogeneous transmission of HIV and other sexual diseases ${ }^{8}$.

If each farm has a susceptibility $\sigma$ and a reproductive potential $\rho$ (transmission rate $x$ infectious period), then the naive homogenous approach (equivalent to assuming that all farms are identical) would suggest that the basic reproductive ratio and critical vaccination $\left(V_{C}\right)$ level necessary for eradication are:

$$
R_{0}^{1}=\langle\rho\rangle\langle\sigma\rangle \approx 1.25 \rightarrow V_{\mathrm{C}}^{1}=1-\frac{1}{\langle\rho\rangle\langle\sigma\rangle} \approx 20 \%
$$

Here the value between angle brackets denotes the average value of a quantity across all farms, and the approximate values come from the estimated parameters from the 2001 epidemic in Great Britain and the 2000 farm census data, and ignore spatial heterogeneity. A more complete analysis shows that the basic reproductive ratio is generally larger (as susceptibility and reproductive potential are expected to be positively correlated), and correspondingly the level of vaccination needed is also increased,

$$
R_{0}^{2}=\langle\rho \sigma\rangle=\langle\rho\rangle\langle\sigma\rangle+\operatorname{cov}_{\rho \sigma} \approx 3.60 \rightarrow V_{\mathrm{C}}^{2}=1-\frac{1}{\langle\rho \sigma\rangle} \approx 72 \%>V_{\mathrm{C}}^{1}
$$

where $\operatorname{cov}_{\rho \sigma}$ is the covariance between $\rho$ and $\sigma$. However, this higher vaccination threshold assumes that the vaccine is evenly distributed across all farms irrespective of their size. A far lower eradication threshold is achieved if vaccination is targeted; a better but not necessarily optimal policy is to vaccinate farms of type i with probability

$$
V_{i}^{3}=\left\{\begin{array}{cl}
1-\frac{1}{\rho_{i} \sigma_{i}} & \text { if } \rho_{i} \sigma_{i}>1 \\
0 & \text { otherwise }
\end{array} \rightarrow\left\langle V^{3}\right\rangle \approx 18 \%<V_{C}^{1}\right.
$$

This formulation works by reducing the fraction of susceptibles in those groups that are most responsible for transmitting infection. This structured population approach can also be extended to examine mass vaccination in the case where the reduction in susceptible numbers is due primarily to immunization rather than infection or culling. The number of expected cases, $I$, can be approximated by $I(t+1) \approx I(t) \exp (\langle\tau \sigma\rangle-g)$ where $\tau$ is the transmission rate, $\sigma$ is the susceptibility, $g$ is the slaughter rate of infected livestock, and all three parameters vary to mimic the slaughtering and vaccination policy. The graph below shows the results of 100 stochastic simulations of the full spatial model, with IP culls and mass vaccination (at 300,000 cattle per day), together with the results of the simple model, which has been parameterized to capture the vaccination of largest cattle farms first (see Supplementary Information). These very different models show a considerable degree of correspondence, although this is far from complete.

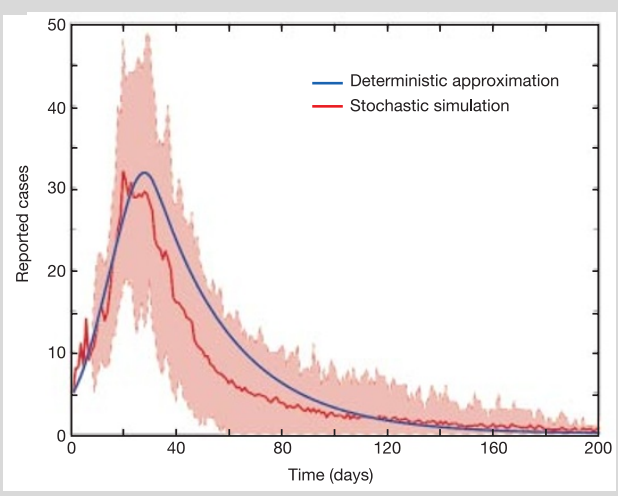


are key to preventing the tail. Thus, taking into account caveats about FMD strain variation as well as the need for effective vaccines ${ }^{7}$ and the logistics to deliver them (Supplementary Information), prompt mass reactive vaccination of cattle on a large scale could significantly reduce the size and duration of future, disseminated FMD epidemics in Great Britain.

The results discussed above assume effective DC and IP culling in addition to vaccination. The dashed lines in Fig. 3 give the equivalent results assuming no DC culling — this leads to a significantly larger and longer epidemic. There are two components to this effect. First, the initial spread of infection before the start of vaccination is greater if DCs are not culled; second, DC culling is important for reducing $R$ in the unvaccinated sheep population, as well as in unprotected cattle. The latter effect depends on the exact value of $R$ in sheep (and any decisions about sheep vaccination); however, these results underline the importance of effective identification and removal of high-risk premises.

For the neighbourhood vaccination approach, we first consider ring vaccination ${ }^{22}$ of cattle, again assuming $90 \%$ efficacy (see Methods). Figure 4a shows epidemic size as a function of the number of vaccinated farms, which itself varies with the size of the vaccinated ring. Ring vaccination generates some benefit, but even $10-\mathrm{km}$ rings only reduce the epidemic size by around $20 \%$. This lack of effectiveness occurs for the following reasons. First, the delay from infection to reporting and from vaccination to protection means that many local cases arise before vaccination has any effect. Second, the localized nature of 'IP-centred' ring vaccination means that neighbouring uninfected areas retain high levels of susceptibility, which can generate new epidemics by means of long-range 'sparks' of infection. Ring vaccination is also much less efficacious if combined with CP culling (red), because the latter removes potentially infected and at-risk premises much more rapidly than local vaccination. Further results (see Supplementary Information) indicate that ring vaccination has relatively little effect on the epidemic tail—again, because it leaves susceptible areas where the infection can percolate.

The final approach of reactive vaccination that we consider is predictive vaccination. Limited ring vaccination does not have a strong effect on either the main body or the tail of the epidemic. Only prompt culling or mass vaccination can 'get ahead' of the main outbreak; however, if the latter strategies were difficult to implement, a shortening of the epidemic tail (with concomitant economic benefits) could in principle be achieved using a 'smarter' predictive vaccination strategy which targets high risk premises.

We demonstrate this potential with the following strategy (see

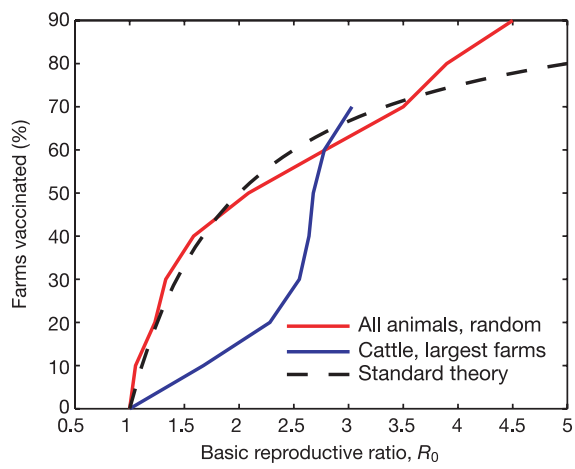

Figure 2 Comparison between simulation (red and blue lines) and standard theory (dashed line) for the critical level of vaccination needed to prevent an epidemic as the basic reproductive ratio, $R_{0}$, varies. Initial conditions are as in Fig. 1. The simulation lines show the level of vaccination needed such that, once movement restrictions are enforced, the average rate of change in the number of cases is zero and hence $R=1$. Different values of $R_{0}$ are modelled by changing all the species-specific transmission rates.
Methods and Box 2). Consider a central 'source' farm in which infection has just been detected (Box 2). We use a subsidiary model to predict the probability that a given farm will be infected from this source in the next disease generation and, on the basis of this, the probability of 'second generation' infection on each farm. As vaccination takes roughly a disease generation to become effective, first generation farms are not the optimal target as they are already likely to be infected. Instead we should focus control in this predictive horizon on those farms most at risk in the second generation. Beginning vaccination 26 days after the detection of FMD, the predictive strategy gives relatively little benefit in terms of the overall epidemic size compared with ring vaccination (Fig. $4 \mathrm{~b}$ ); however, the duration of the epidemic can be significantly shortened by the predictive strategy, whereas ring vaccination has much less effect (Fig. 4c). This is further demonstrated in Fig. 5a, where expected epidemic curves show that predictive vaccination of just 100 farms per day significantly truncates the epidemic, whereas the addition of CP culling lowers the total epidemic size. Figure $5 \mathrm{~b}$ shows that if predictive vaccination were applied earlier ( 8 days after detection) then it also has the potential to significantly lower the deterministic component of the epidemic, whereas even late uptake of vaccination ( 40 days after detection) can still reduce the duration of the tail. Note that even stringent DC and CP culling cannot achieve this truncation of the outbreak ${ }^{7}$.
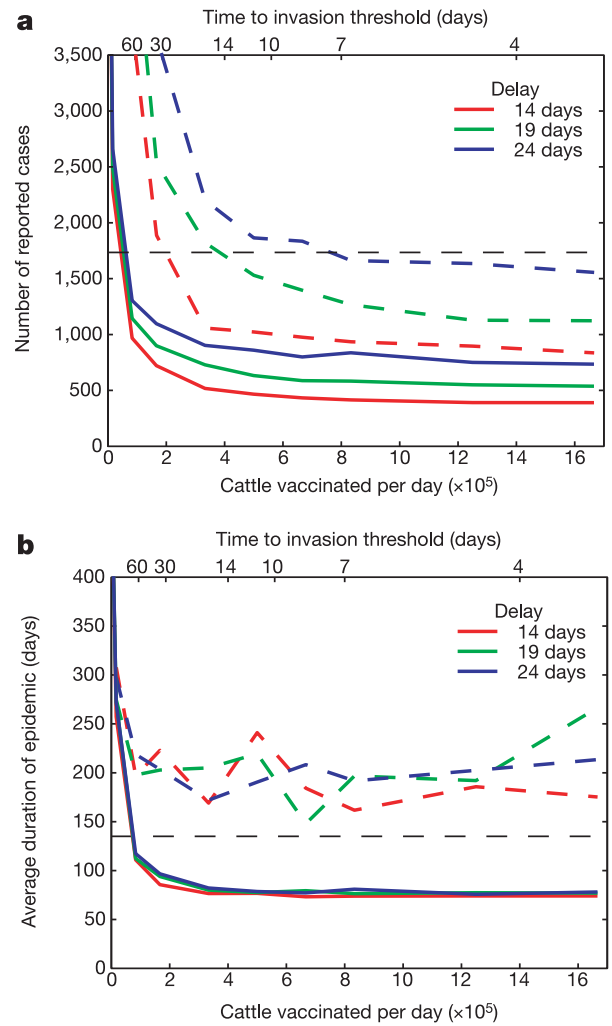

Figure 3 Graph to show how an epidemic can be controlled by the rapid vaccination of cattle during the early stages, using the 2001 epidemic of Great Britain as a template. Throughout, vaccination is of cattle only and assumed to be at $90 \%$ efficacy-initially large cattle farms are targeted, as these are most at risk. a, Expected number of farms reporting infection against the number of cattle vaccinated per day (bottom axis) or the corresponding time to achieve the disease eradication threshold of around 5.5 million cattle (top axis). Solid lines show the result when IP and DC controls are used; dashed lines show the result when only IP culling is performed. The horizontal line gives the expected number of cases if $\mathrm{CP}$ culling is also used but no vaccination is performed. Three different patterns are simulated corresponding to different delays between the detection of the disease and the start of protection by vaccination. $\mathbf{b}$. The expected duration of the epidemic for the six patterns investigated in $\mathbf{a}$. 


\section{Discussion}

Our central findings concerning levels of herd immunity and the potential impact of predictive vaccination seem robust to key model assumptions and the limitations of the input data (see Supplementary Information). However, as outlined below (and discussed in the Supplementary Information), these conclusions must confront additional caveats before they can inform policy. Key areas for discussion are: (1) trade issues and cooperation from livestock owners; (2) the practical consequences of vaccination for clinical surveillance, follow-up vaccination to protect newborn animals, vaccination of sheep or pigs in addition to cattle, and the control implications of seasonality in livestock husbandry; and (3) the key issue of understanding the spatial spread of infection (which will vary both with the pathogen strain, its host specificity, farming
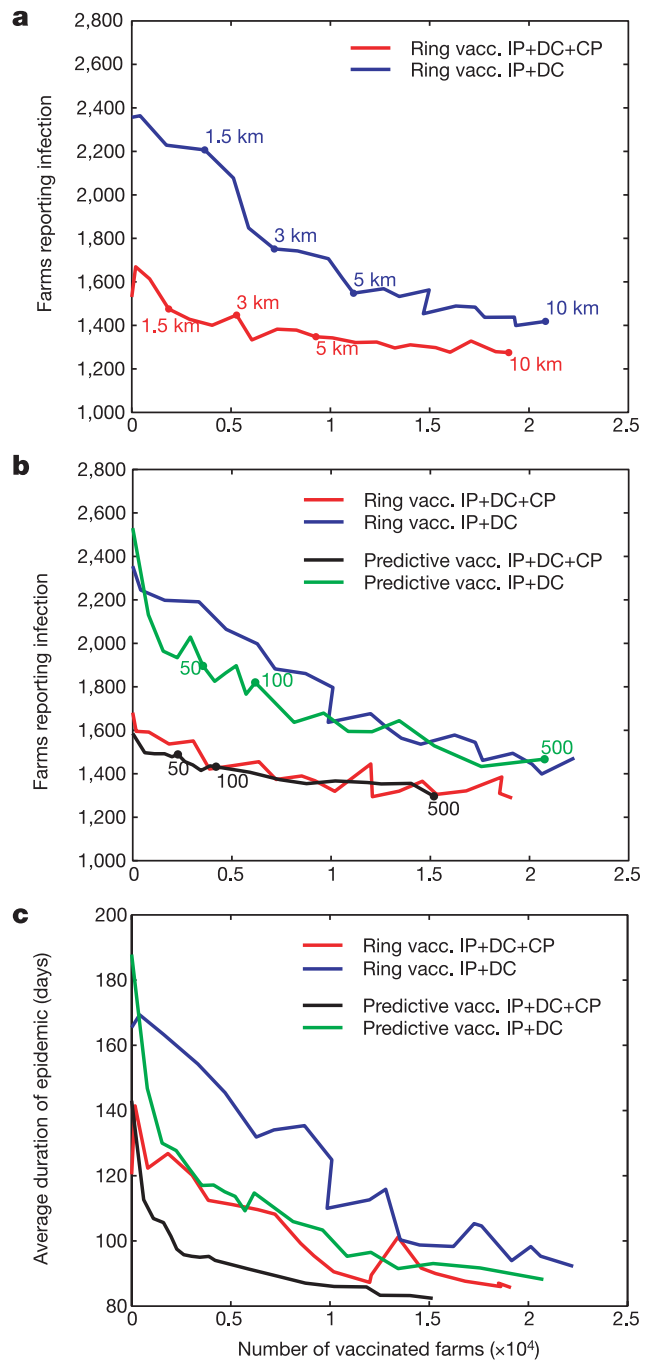

Figure 4 Results from predictive vaccination in comparison to ring vaccination and other control measures. Initial conditions come from the 2001 epidemic in Great Britain, and in general vaccination begins 26 days after disease detection (equivalent to 18 March in the 2001 epidemic). Two different culling policies are considered, either culling DCs and CPs at the observed temporally varying levels ${ }^{7}$, or culling DCs only where the culling level rapidly rises to one $\mathrm{DC}$ per IP. a, The expected number of farms reporting infection against the total number of farms vaccinated during the epidemic. All non-culled farms within a fixed radius of the IP are vaccinated. Radii of 1.5, 3, 5 and $10 \mathrm{~km}$ are indicated. $\mathbf{b}$, As for a, but with the results for predictive vaccination superimposed. Each day a fixed number of the most at-risk farms are vaccinated; vaccinating levels of 50, 100 and 500 per day are indicated. c, Comparison between ring and predictive vaccination in terms of the average duration of the epidemic. practices and the spatial distribution and movement of livestock).

The prophylactic vaccination results mirror a basic epidemiological tenet ${ }^{8}$ — raising regional herd immunity sufficiently to reduce the effective reproductive ratio, $R$, below unity will protect from major epidemics of FMD. Given the observed reproductive ratio of the disease after the imposition of movement restrictions in Great Britain in 2001, immunization of more than 30\% of farms would achieve this effect; moreover, targeted vaccination of at-risk groups, such as large cattle farms, could be more effective per immunization. We stress, however, that the prophylactic herd immunity result does not take account of the problem of strain variation in FMD.

Mass reactive vaccination once FMD has been detected and the strain identified could also be effective. Such control would require a rapid and high uptake of vaccination, necessitating cooperation from farmers, and considerable logistical effort and preparation (see Supplementary Information). However it also depends on four other factors. First, a movement ban needs to be instigated promptly, to minimise dissemination of the infection and reduce its reproductive ratio ${ }^{23}$. Second, effective detection and removal of livestock on at-risk premises is an important adjunct to vaccination. Third, the birth of large numbers of cattle and sheep at particular times of the year may mean that supplementary vaccination campaigns become necessary. Last, more transmissible strains of FMD virus would require a much greater effort.

The main problem for reactive vaccination is the delay before protection ${ }^{14,22}$. This particularly affects limited ring vaccination, where the infection spreads to local premises before the vaccination can protect them, allowing 'escape' from the edge of the ring. Predictive vaccination is the most efficient limited reactive strategy;

\section{Box 2 \\ Predictive vaccination}

Schematic diagrams showing the probability of being infected in each generation. In generation 0 the central farm is infected (it is an IP), whereas the surrounding farms are assumed susceptible. At the end of generation 1, the animals in the IP show signs and the farm reports infection - at this stage vaccination should occur. In generation 1, farms are infected in relation to their distance from the IP. The two farms that are closest have very high probabilities of already being infected, and therefore are unlikely to be infected in the next generation. It is the cluster of farms (circled blue) that have the greatest chance of being infected in generation 2 because: there is a 99\% chance that at least one of them is still susceptible in generation 1 ; they can get infected in generation 2 from farms close to the original IP; and there is a $49 \%$ chance that at least one of them got infected in generation 1 , subsequent spread to the remainder in generation 2 is then likely. Therefore, in response to the IP, the circled cluster of farms should be vaccinated with the highest priority as here the vaccine has the maximal effect - vaccinating the nearest farms would be futile as they will already be infected before the vaccine takes effect.

The efficacy of predictive vaccination depends on how reliably risk factors for infection and transmission can be identified. Here we have assumed complete knowledge of those risk factors (although the actual outcome is still stochastic in nature). The effectiveness of the strategy will be less if risk factors are less well known.

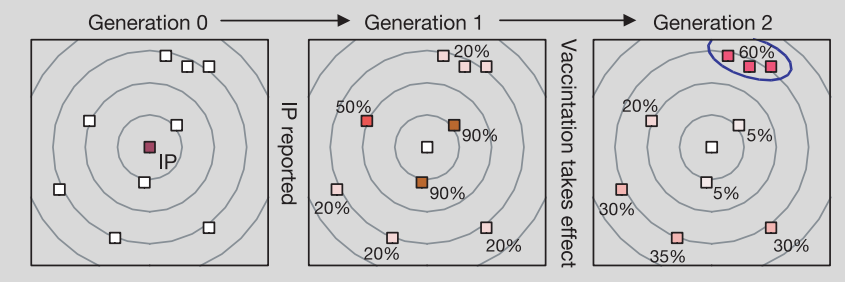



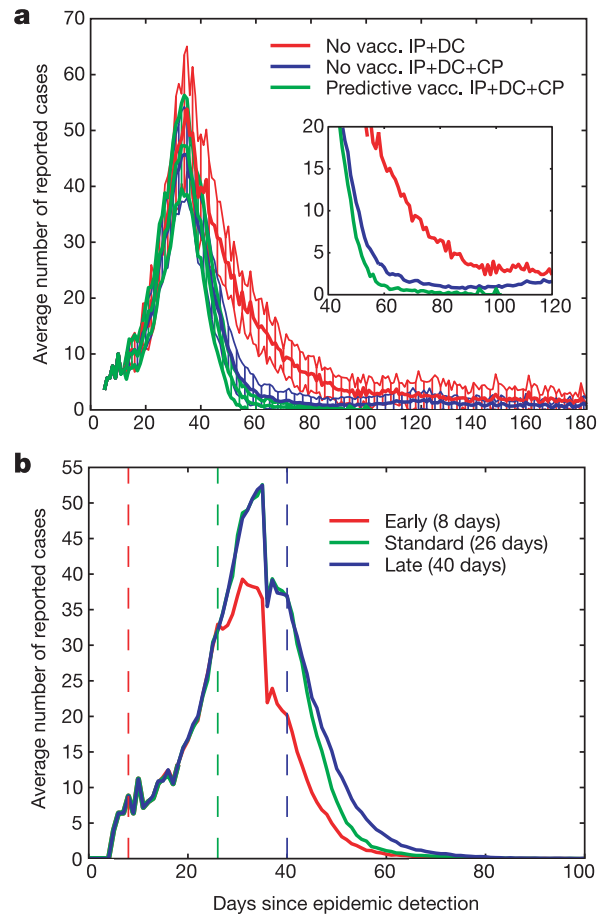

Figure 5 Effects of predictive vaccination on the average epidemic profile, using the same initial conditions as in Fig. 4. a, Comparison between culling DCs only, culling DCs and CPs, and culling DCs and CPs in addition to predictive vaccination at a rate of 100 farms per day. Solid lines show the average number of daily cases, with two standard deviations indicated. The inset shows the period around the start of the epidemic tail in greater detail. b. The effect of starting predictive vaccination at different dates. The usual start date of 26 days after detection is shown in green, and this is compared to an earlier start of day 8 (red) and a later start of day 40 (blue).

it is essentially an extension of the targeting of at-risk farms with spatial clustering of susceptible farms and the location of infection added as risk factors (Box 2). The method works by blocking transmission to high-risk second-generation nodes in the farm network, potentially reducing the tail of simulated outbreaks by many months. This strategy needs greater epidemiological input before it could be applied: first, to refine our appreciation of risk factors for infection; second, although the method essentially identifies the most at-risk farms, this information then needs to be combined with local expertise to direct the distribution of vaccination.

The optimal strategy depends critically on the initial dissemination of the epidemic. Our analysis is based on the relatively pessimistic (and therefore conservative) model of a highly disseminated epidemic; consequently, we predict that a widespread mass vaccination campaign would be necessary to prevent a major epidemic. Clearly, a single point source epidemic might permit a more limited regional response by ring vaccination; however, our results stress the importance of increasing herd immunity over a relatively wide area to inhibit stochastic jumps of infection for a given spatial transmission kernel.

More broadly, our results probe the robustness of simple mathematical models for eradication of disease by mass regional or national vaccination campaigns ${ }^{8}$. Efficient mass vaccination effectively damps down the spread of infection at all scales; we show that, even in a complex spatial landscape, this effect can be approximated by standard 'mass action' models. However, this correspondence only applies to the threshold for eradication of infection by vaccination: lower levels of vaccination can generate complex, nonlinear, spatio-temporal disease dynamics. The pattern here is provided by endemic childhood infections, such as measles and pertussis ${ }^{8}$, where the interaction between vaccination, epidemic dynamics, seasonal forcing and metapopulation structure can generate a range of subtle dynamical effects ${ }^{24-26}$.

Turning to local control, this is the first exploration, to our knowledge, of predictive vaccination for optimal control of an infection in a complex spatial landscape. Moreover, our conclusions contradict those obtained using much simpler models for ring vaccination $^{27}$. The approach will repay further analytical work to optimise control for a given transmission kernel shape and spatial distribution of hosts - a particularly interesting question here is how to optimally reduce the 'small world' nature of rare long-range dispersal, which can spark off damaging new local outbreaks during the tail of the epidemic. The spatial focusing of vaccination effort also mirrors, in a more quantitative context, empirical strategies for identifying at-risk individuals in human vaccination campaigns ${ }^{8}$.

There is considerable scope for the development of predictive strategies in the control of emergent human and animal infections. We stress that a prerequisite for success is collaboration between empirical and theoretical researchers and field epidemiologists to have suitable models and relevant databases in place as much as possible before disease outbreaks begin.

\section{Methods}

The basic model

The model used throughout this paper is a spatial stochastic simulation, where the infectious state of every livestock farm in Britain is predicted on a daily basis. The rate, $r$, at which farm $\mathrm{i}$ (which is currently susceptible) is infected is given by,

$$
R_{\mathrm{i}}=\sum_{L \in \text { livestock }} S_{L} N_{L}^{\mathrm{i}} \times \sum_{j \in \text { infectious }} \sum_{L \in \text { livestock }} T_{L} N_{L}^{\mathrm{j}} \times K\left(d_{\mathrm{ij}}\right)
$$

where $N_{L}^{\mathrm{i}}$ is the number of livestock of type $L$ within farm i; $S_{L}$ is the susceptibility of livestock $L ; T_{L}$ is the transmission rate of livestock $L ; d_{\mathrm{ij}}$ is the distance between farms $\mathrm{i}$ and $\mathrm{j}$; and $K$ is the transmission kernel. Once infected, farms are assumed to remain in an exposed (but not infectious) state for 4 days, after which they become infectious and can transmit the virus to other farms. Nine days after infection, after the appearance of clinical signs, the presence of the disease is reported; after a further delay of between 1 and 3 days (depending of the stage of the epidemic) the animals on the infected farm are slaughtered and the appropriate neighbourhood cull is performed (see Supplementary Information). It was estimated that around $40 \%$ of dangerous contacts were infected. More details of the parameter estimation and model validation can be found elsewhere ${ }^{7}$.

\section{Prophylactic vaccination}

Prophylactic vaccination is implemented by reducing the number of livestock in those farms vaccinated. In the simplest model, farms are selected at random for vaccination and the outcome is determined independently for each animal. As such the number of susceptible animals remaining in a farm after vaccination is given by a binomial distribution. When vaccinating cattle only, those farms without cattle are ignored. A targeted model is also simulated, where the cattle on those farms with the most cattle are vaccinated first.

Simulations are started with the infection of five randomly chosen farms; often these farms may be quite small and in isolated areas so that the disease dies out. The infection is allowed to spread unchecked for two weeks, after which time movement controls and culling measures are implemented.

In addition to vaccination, all models assume that IPs are culled within $24 \mathrm{~h}$. In most of the simulations culling of DCs is also performed, this is assumed to occur 2 days after reporting, and within one month asymptotes to a ratio of one DC culled per IP. Finally, all simulations are compared to the situation where vaccination is not implemented, but CP culling is; the level of CP culling mimics that achieved during the 2001 epidemic in Great Britain.

\section{Reactive vaccination}

Reactive vaccination is implemented using the 2001 epidemic in Great Britain as a template. All simulations start 3 days after the detection of the disease (equivalent to the 23 February in the 2001 epidemic, when movement restrictions were enforced), and use the estimated distribution of infection at this time as initial conditions $\mathrm{s}^{23}$. The timing and level of culls also follows the 2001 pattern. Vaccination is applied to cattle only and is optimistically assumed to offer protection after 4 days; those farms infected before protection is complete were assumed to progress through the normal sequence of infection. Mass vaccination is begun between 1 week and 17 days after the detection of cases. Limited vaccination is generally assumed to start 26 days after detection (equivalent to 18 March in the 2001 epidemic, when it was realized that stronger control measures were needed), and occurs in response to an infected premise 2 days after it is reported.

For the ring vaccination approach, all those farms within a fixed distance of an infected premise will be vaccinated. Note that owing to the nature of the census data this is taken as the distance between farmhouses, rather than the distance between livestock holdings. Vaccinating within the ring was assumed to occur 2 days after the triggering IP is reported, with the entire ring being vaccinated within $24 \mathrm{~h}$ (although a further 4 days is needed 
before protection is achieved); this is clearly an optimistic assumption and will depend on local logistical constraints.

For the predictive vaccination approach, a fixed number of farms are vaccinated each day, these are chosen to minimise the number of subsequent cases. Using the farms reporting infection so far, we can approximate the infectious state of the system 9 days ago. This is only an approximation, as those farms that are infected but culled before clinical signs arise are not recognized as potential sources of infection. Each week, multiple simulations of a subsidiary model use the reported cases so far to calculate the probability that each susceptible farm becomes infected between 6 and 13 days time. Those farms infected within 6 days would not be protected by vaccination, whereas those farms infected after 13 days could be protected by next week's vaccination. Therefore the prediction procedure requires multiple replicates of a 22 -day ( 9 plus 13) period to determine the infection probability. The farms are then ranked according to the expected decrease in transmission that would be caused by vaccination of their cattle herds.

Received 17 June; accepted 4 December 2002; doi:10.1038/nature01343.

Published online 22 December 2002.

1. Woolhouse, M. \& Donaldson, A. Managing foot-and-mouth-The science of controlling disease outbreaks. Nature 410, 515-516 (2001).

2. Gibbens, J. C. et al. Descriptive epidemiology of the 2001 foot-and-mouth disease epidemic in Great Britain: the first five months. Vet. Record 149, 729-743 (2001).

3. Ferguson, N. M., Donnelly, C. S. \& Anderson, R. M. Transmission intensity and impact of control policies on the foot and mouth epidemic in Great Britain. Nature 413, 542-548 (2001).

4. Woolhouse, M. et al. Foot-and-mouth disease under control in the UK. Nature 414, 258 (2001).

5. Morris, R. S., Wilesmith, J. W., Stern, M. W., Sanson, R. L. \& Stevenson, M. A. Predictive spatial modelling of alternative control strategies for the foot-and-mouth disease epidemic in Great Britain, 2001. Vet. Record 149, 137-144 (2001).

6. Ferguson, N. M., Donnelly, C. S. \& Anderson, R. M. The foot-and-mouth epidemic in Great Britain pattern of spread and impact of interventions. Science 292, 1155-1160 (2001).

7. Keeling, M. J. et al. Dynamics of the 2001 UK foot and mouth epidemic: stochastic dispersal in a heterogeneous landscape. Science 294, 813-817 (2001).

8. Anderson, R. M. \& May, R. M. Infectious Diseases of Humans (Oxford Science, Oxford, 1991).

9. Pastoret, P. P. et al. (eds) Veterinary Vaccinology (Elsevier, Amsterdam, 1997).

10. McLean, A. R. \& Blower, S. M. Modelling HIV vaccination. Trends Microbiol. 3, 458-463 (1995).

11. Woolhouse, M. E. J., Haydon, D. T. \& Bundy, D. A. P. The design of veterinary vaccination programmes. Vet. J. 153, 41-47 (1997).

12. Williams, J. R., Nokes, D. J. \& Anderson, R. M. Targeted hepatitis B vaccination-a cost effective immunisation strategy for the UK? J. Epidemiol. Comm. Health 50, 667-673 (1996).

13. Woolhouse, M. E. J. et al. Failure of vaccination to prevent outbreaks of foot-and-mouth disease. Epi. Infect. 116, 363-371 (1996).

14. Barnett, P. V. \& Carabin, H. A review of emergency foot-and-mouth disease (FMD) vaccines. Vaccine 20, 1505-1514 (2002).

15. EU strategy for emergency vaccination against foot-and-mouth disease (FMD). Report of the Scientific Committee on Animal Health and Animal Welfare 〈http://europa.eu.int/comm/dg24/ health/sc/scah/index_en.html $>$ (1999).

16. Woolhouse, M. E. J. \& Bundy, D. A. P. in Veterinary Vaccinology (eds Pastoret, P. P. et al.) 565-573 (Elsevier, Amsterdam, 1997).

17. Woolhouse, M. E. J. et al. Heterogeneities in the transmission of infectious agents: implications for the design of control programs. Proc. Natl Acad. Sci. USA 94, 338-342 (1997).

18. HMSO Northumberland Report. The Report of the Committee of Inquiry on Foot and Mouth Disease 1968 (HMSO, London, 1968).

19. Howard, S. C. \& Donnelly, C. A. The importance of immediate destruction in epidemics of foot and mouth disease. Res. Vet. Sci. 69, 189-196 (2000).

20. Yang, P. C. et al. Epidemiological characteristics and financial costs of the 1997 foot-and-mouth disease epidemic in Taiwan. Vet. Record 145, 731-734 (1999).

21. Donaldson, A. I. et al. Relative risks of the uncontrollable (airborne) spread of FMD by different species. Vet. Record 148, 602-604 (2001).

22. Salt, J. S. et al. in Veterinary Vaccinology (eds Pastoret, P. P. et al.) 641-652 (Elsevier, Amsterdam, 1997).

23. Haydon, D. T. et al. The construction and analysis of epidemic trees with reference to the $2001 \mathrm{UK}$ foot-and-mouth outbreak. Proc. R. Soc. Lond. B (in the press); published online (doi:10.1098/ rspb.2002.2191).

24. Keeling, M. J., Rohani, P. \& Grenfell, B. T. Seasonally-forced disease dynamics explored as switching between attractors. Physica D 148, 317-335 (2001).

25. Rohani, P., Earn, D. J. D. \& Grenfell, B. T. Opposite patterns of synchrony in sympatric disease metapopulations. Science 286, 968-971 (1999).

26. Grenfell, B. T., Biørnstad, O. N. \& Kappey, J. Travelling waves and spatial hierarchies in measles epidemics. Nature 414, 716-723 (2001).

27. Muller, J., Schonfisch, B. \& Kirkilionis, M. Ring vaccination. Math. Biol. 41, 143-171 (2000).

28. Keeling, M. J. The effects of local spatial structure on epidemiological invasions. Proc. R. Soc. Lond. B 266, 859-869 (1999).

Supplementary Information accompanies the paper on Nature's website ( http://www.nature.com/nature).

Acknowledgements This research was supported by the Royal Society (M.J.K.) and the Wellcome Trust (M.E.J.W. and B.T.G.). Data were provided by the Department of the Environment, Food and Rural Affairs, the Scottish Executive Rural Affairs Department, and the National Assembly for Wales Statistical Directorate. We thank D. Shaw, S. St Rose, M. Chase-Topping, D. Haydon and L. Matthews for their help.

Competing interests statement The authors declare that they have no competing financial interests.

Correspondence and requests for materials should be addressed to M.J.K. (e-mail: m.j.keeling@warwick.ac.uk). 